\title{
SIMULATIONS OF NOISE-FREE STOCHASTIC RESONANCE IN SPIN-WAVE CHAOS
}

\author{
A. Krawiecki ${ }^{a}$, E. Reibold $^{d}$, H. BenneR ${ }^{d}$ AND A. Sukiennicki ${ }^{a, b}, c$ \\ ${ }^{a}$ Institute of Physics, Warsaw University of Technology \\ Koszykowa 75, 00-662 Warsaw, Poland \\ ${ }^{b}$ Department of Solid State Physics, University of Łódź \\ Pomorska 149/153, 90-283 Łódź, Poland \\ ${ }^{c}$ Institute of Physics, Polish Academy of Sciences \\ Al. Lotników 32/46, 02-668 Warsaw, Poland \\ ${ }^{d}$ Institut für Festkörperphysik, Technische Universität Darmstadt \\ Hochschulstraße 6, 64289 Darmstadt, Germany
}

Numerical simulations of noise-free stochastic resonance and aperiodic stochastic resonance in chaotic ferromagnetic resonance are presented. The model, based on three-magnon interactions between the externally excited uniform mode and pairs of spin waves, shows on-off intermittency. The rf magnetic field amplitude is slowly modulated by a small periodic or aperiodic signal, and the output signal, which reflects the occurrence of laminar phases and bursts in the time series of spin-wave amplitudes, is analyzed. On variation of the dc magnetic field the signal-to-noise ratio of the output signal and the correlation function between modulation and output signal pass a maximum, which indicates the occurrence of periodic and aperiodic stochastic resonance, respectively. The role of thermal magnon excitations in the occurrence of this maximum is clarified. The results are compared with experimental findings obtained in other types of intermittency.

PACS numbers: 76.50.+g, 75.30.Ds, 05.45.+b, 05.40.+j

Stochastic resonance (SR) occurs in systems driven by a coherent signal and noise where the noise intensity can be tuned in a way to maximize the coherence of a properly chosen response signal [1]. The coherent signal is usually periodic. In the case of an aperiodic signal the term "aperiodic stochastic resonance" (ASR) is used [2]. SR appears in systems with two (or more) distinguishable states (e.g. in bistable systems), in which the probability to find the system in one of these two states is modulated by the coherent signal [3]. SR occurs due to the matching of two time scales: the (mean) modulation frequency and the noise-controlled switching rate between the two states. In chaotic systems, so-called "noise-free SR" can be observed without external noise by tuning the chaotic dynamics through the control parameters [4]. E.g. by varying one of the control parameters in systems with intermittency one changes the mean duration of laminar phases and bursts, 
which play the role of the two states of the system, and tunes it to the (mean) period of coherent modulation. Noise-free SR was first observed experimentally in chaotic ferromagnetic resonance (FMR) [5], and numerical simulations of the corresponding model were presented in Ref. [6].

In this paper we consider a numerical model of noise-free SR in the "coincidence regime" of the first-order Suhl instability [7]. The uniform mode driven by the rf field of frequency $\omega$ (close to its eigenfrequency $\omega_{0}$ ) decays into pairs of spin waves (SW) with opposite wave vectors and frequencies $\omega_{k} \approx \omega / 2$, as soon as the rf field amplitude $h_{T}$ exceeds the threshold $h_{T}^{\text {thr }}$. In our model the rf field amplitude is slowly modulated with a signal of frequency $\omega_{s} \ll \omega$, and only two SW pairs are included to interact with the uniform mode. The equations of motion for the weakly time-dependent complex amplitudes of the uniform mode $a_{0}$ and of the spin waves $a_{1}$ and $a_{2}$ read [6]

$$
\begin{aligned}
& \dot{a}_{0}=\left|\delta_{0}\right|\left|\delta_{1}\right| \varepsilon\left(t^{\prime}\right)-\left(\eta_{0}+\mathrm{i} \Delta \omega_{0}\right) a_{0}-\mathrm{i} a_{1}^{2}-\mathrm{i}\left(\left|V_{0,2}\right| /\left|V_{0,1}\right|\right) a_{2}^{2}+\xi_{\text {th }}, \\
& \dot{a}_{1}=-\left(\eta_{1}+\mathrm{i} \Delta \omega_{1}\right) a_{1}-\mathrm{i} a_{1}^{*} a_{0}+\xi_{\text {th }}, \\
& \dot{a}_{2}=-\left(\eta_{2}+\mathrm{i} \Delta \omega_{2}\right) a_{2}-\mathrm{i}\left(\left|V_{0,2}\right| /\left|V_{0,1}\right|\right) a_{2}^{*} a_{0}+\xi_{\text {th }},
\end{aligned}
$$

where $\eta_{0, k}, k=1,2$ are phenomenological damping parameters, $\Delta \omega_{0}=\omega_{0}-\omega$, $\Delta \omega_{k}=\omega_{k}-\omega / 2, \delta_{0, k}=\eta_{0, k}+\mathrm{i} \Delta \omega_{0, k}, \varepsilon=h_{T} / h_{T}^{\text {thr }}, V_{0, k}$ are the respective three-magnon coupling coefficients and $\xi_{\text {th }}$ is the level of thermal excitation of SW. In (1) all detunings, dampings, and amplitudes are dimensionless and normalized to $\eta_{1}$, and the dot denotes the derivative with respect to rescaled time $t^{\prime}=\eta_{1} t$. - In real systems we have $\eta_{1} \simeq 10^{6} \mathrm{~s}^{-1}$ and $\xi_{\text {th }} \simeq 10^{-10}$ at the liquid helium temperature [6]. Using the following parameters in our simulations: $\eta_{0}=1.25$, $\eta_{1} \equiv 1.0, \eta_{2}=0.8, \Delta \omega_{0}=-1.5, \Delta \omega_{1}=3.0, \Delta \omega_{2}=2.62,\left|V_{02}\right| /\left|V_{01}\right|=0.754[8]$, the system (1) becomes chaotic for $\varepsilon>1.95$. For $\varepsilon<\varepsilon_{c} \approx 3.02$ we found on-off intermittency [9] characterized by a sequence of laminar phases, during which $\left|a_{2}\right| \approx 0$, and by chaotic bursts (Fig. 1) [8]. For $\varepsilon>\varepsilon_{c},\left|a_{2}\right|$ decays to zero. Similar behaviour was experimentally observed in chaotic FMR [10].

Assuming that the coherent signal is applied through amplitude modulation of the driving $\mathrm{rf}$ field we set $\varepsilon\left(t^{\prime}\right)=\varepsilon_{0}+A f\left(t^{\prime}\right)$, where $\varepsilon_{0}=3.01$ and $A=0.15$. The function $f\left(t^{\prime}\right)$ is either periodic in time, $f\left(t^{\prime}\right)=\sin \left(\omega_{s} t^{\prime}\right)$, or aperiodic, $f\left(t^{\prime}\right)=0$ or 1 , where each of the two values is chosen at random every $\Delta t_{s}^{\prime}=200$. In contrast to Ref. [6], where $\varepsilon_{0}$ was used as a control parameter, here we chose the dc magnetic field. Its variation results in a detuning of the eigenfrequencies and corresponds in (1) to changes of $\Delta \omega_{0}$ and $\Delta \omega_{k}$. In order to model noise-free SR or ASR in chaotic SW dynamics, we solved Eq. (1) numerically on variation of $\Delta \omega_{0}$ (and equal variation of $\left.\Delta \omega_{1}, \Delta \omega_{2}\right)$. The resulting time series for $\left|a_{2}\right|$ still shows on-off intermittency (Fig. 1). To obtain a more distinct separation of both phases we analyzed the distorted signal $y\left(t^{\prime}\right)=\Theta\left(\left|a_{2}\right|-\theta\right)$, where $\Theta(\cdot)$ is the Heaviside unit step function and $\theta=0.1$ is an arbitrary but reasonable threshold for the burst. Hence $y\left(t^{\prime}\right)$ reflects the sequence of laminar and burst phases in the time series of $\left|a_{2}\right|$. It turns out that the mean duration of both phases is changed on variation of the SW detunings. Since it is also modulated by $A f\left(t^{\prime}\right)$, the matching of the two time scales necessary for noise-free SR can occur for the properly chosen $\Delta \omega_{0}$. 

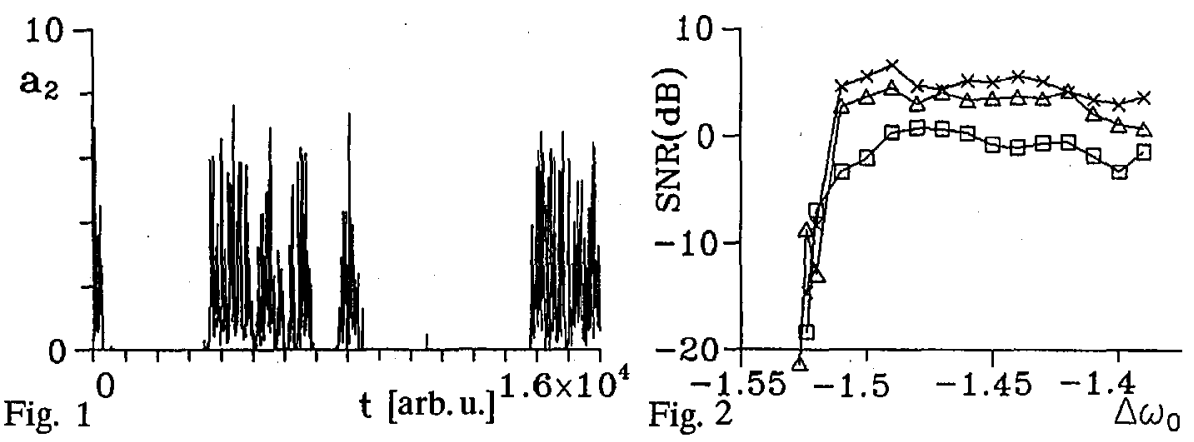

Fig. 1. Time series of $\left|a_{2}\right|$ for $\Delta \omega_{0}=-1.5,2 \pi / \omega_{s}=128$.

Fig. 2. $S N R$ vs. $\Delta \omega_{0}$ for different values of $2 \pi / \omega_{s}: 16$ (squares), 32 (triangles), 128 (crosses) and for $\xi_{\text {th }}=10^{-10}$.

In order to characterize SR we used typical measures [1, 2]. For periodic $f(t)$ we evaluated the power spectral density $S(\omega)$ of $y(t)$ and calculated the signal-to-noise ratio $(S N R)$ at $\omega=\omega_{s}$ vs. $\Delta \omega_{0}$. For ASR with aperiodic $f(t)$ we evaluated the correlation function $C_{1}$ between $y(t)$ and $f(t)$ vs. $\Delta \omega_{0}$. These measures are defined as follows:

$$
S N R=10 \log \frac{S\left(\omega_{s}\right)-S_{\mathrm{N}}\left(\omega_{s}\right)}{S_{\mathrm{N}}\left(\omega_{s}\right)}, \quad C_{1}=\frac{\langle y f\rangle-\langle y\rangle\langle f\rangle}{\sqrt{\left(\left\langle f^{2}\right\rangle-\langle f\rangle^{2}\right)\left(\left\langle y^{2}\right\rangle-\langle y\rangle^{2}\right)}},
$$

where $S_{N}\left(\omega_{s}\right)$ is the noise background at $\omega_{s}$ in the power spectral density, on which the peak with the height $S\left(\omega_{s}\right)-S_{\mathrm{N}}\left(\omega_{s}\right)$ coming from the periodic component of $y(t)$ is superimposed, and the brackets denote the time average.

The results of our numerical simulations of noise-free SR with periodic modulation $f(t)$ are summarized in Figs. 2, 3, and with aperiodic modulation in Fig. 4.

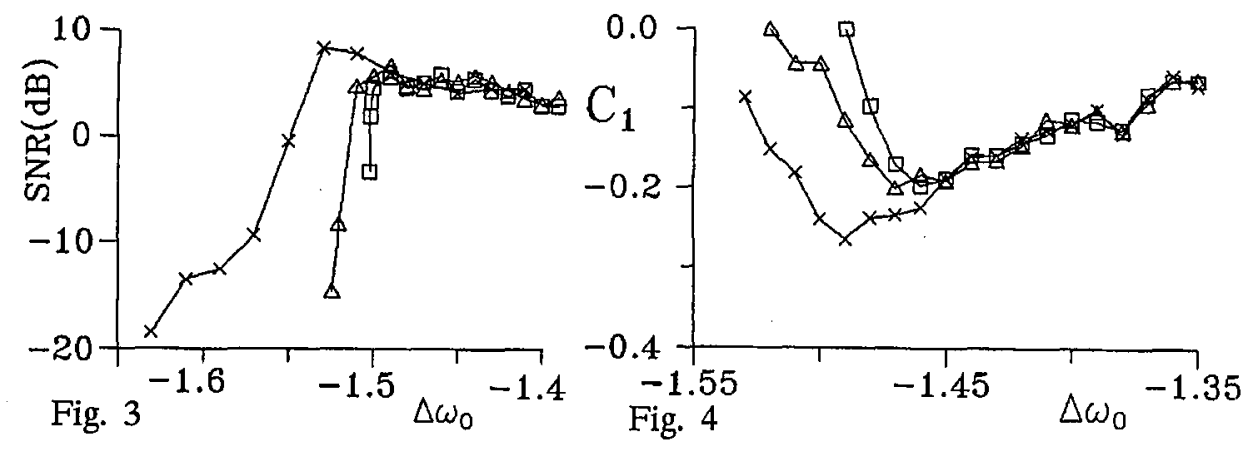

Fig. 3. $S N R$ vs. $\Delta \omega_{0}$ for various $\xi_{\text {th }}: 0$ (squares), $10^{-10}$ (triangles), $10^{-4}$ (crosses, unphysically large) and for $2 \pi / \omega_{s}=128$.

Fig. 4. $C_{1}$ vs. $\Delta \omega_{0}$ for various $\xi_{\text {th }}$ as in Fig. 3. 
In Figs. 2, 3 a maximum of $S N R$ vs. $\Delta \omega_{0}$ can be seen, and in Fig. 4 a minimum (instead of a maximum) of $C_{1}$ vs. $\Delta \omega_{0}$ (the latter appears because bursts of $\left|a_{2}\right|$ occur most frequently when $\varepsilon(t)$ is at a minimum). Such shapes are typical of SR and ASR. In Fig. 2 the characteristic increase in $S N R$ with decreasing $\omega_{s}$ occurs quite markedly (adiabatic limit [3]). In Figs. 3, 4 we have shown the influence of thermal excitation of $\mathrm{SW}$ : the extrema of the $S N R$ or $C_{1}$ curves are broadened and occur more distinctly. Thus, noise-free SR in experiments with on-off intermittency in SW dynamics will appear due to the combined effect of internal chaotic dynamics and weak thermal excitations of SW (the effect of $\xi_{\text {th }}$ in other kinds of intermittency is not necessarily so large [6]).

As an extension of the previous results [6], our present simulations are focussed on the dc magnetic field serving as the control parameter which determines the "level of stochasticity" in noise-free SR, while the coherent signal was applied by modulation of the rf field amplitude. Noise-free stochastic resonance with periodic modulation of the rf field amplitude and variation of the dc field was also observed experimentally in chaotic FMR on a different type of intermittency [5]. The results of our simulations are qualitatively similar to the results of this experiment. Moreover, the modeling was extended to the case of ASR, which suggests that experimental observation of noise-free ASR in intermittent SW dynamics is possible. In chaotic nonlinear FMR, low-dimensional models, though oversimplified, are usually in qualitative agreement with experiment. Thus the results of the present paper, together with those of Refs. [5, 6], show that chaotic nonlinear FMR is a useful tool for the experimental investigation of various kinds of noise-free SR.

\section{References}

[1] L. Gammaitoni, P. Hänggi, P. Jung, F. Marchesoni, Rev. Mod. Phys. 70, 223 (1998).

[2] J.J. Collins, C.C. Chow, A.C. Capela, T.T. Imhoff, Phys. Rev. E 54, 5575 (1996).

[3] B. McNamara, K. Wiesenfeld, Phys. Rev. A 39, 4854 (1989).

[4] V.S. Anischenko, A.B. Neiman, M.A. Safonova, J. Stat. Phys. 70, 183 (1993).

[5] E. Reibold, W. Just, J. Becker, H. Benner, Phys. Rev. Lett. 78, 3101 (1997).

[6] A. Krawiecki, A. Sukiennicki, Acta Phys. Pol. B 30, 2499 (1999).

[7] H. Suhl, J. Phys. Chem. Solids 1, 209 (1957).

[8] A. Krawiecki, A. Sukiennicki, Acta Phys. Pol. A 88, 269 (1995).

[9] J.F. Heagy, N. Platt, S.M. Hammel, Phys. Rev. E 49, 1140 (1994).

[10] F. Rödelsperger, A. Čenys, H. Benner, Phys. Rev. Lett. 75, 2594 (1995). 